\title{
Changing resistance pattern of Shigella isolates over a period of five years
}

\author{
Veenu Gupta, Deepinder Kaur, Manisha Aggarwal, Jaspreet Kaur, Priyam Chawla, Jyoti Chaudhary \\ Department of Microbiology, Dayanand Medical College and Hospital, Ludhiana, Punjab, India.
}

\begin{abstract}
Background and objectives: Diarrheal diseases are an important cause of morbidity and mortality in children in developing countries. Among diarrheagenic agents, Shigella should be emphasized because of its prevalence and the severity of the associated disease. The present study was done to study the prevalence and antimicrobial susceptibility pattern of Shigella isolates in stool. Materials and methods: Stool samples were collected from cases of dysentery and diarrhea in the laboratory from Jan 2009 to Dec 2013. The specimens were processed and inoculated as per standard protocol. The susceptibility of Shigella serogroups to different antibiotics was done as per the Clinical and Laboratory Standards Institute (CLSI) guidelines. Antibiotic susceptibility between different Shigella serogroups was compared and trends of drug resistance to various antimicrobial agents over a period of five years was seen. Results: Of a total of 6117 samples, Shigella serogroups were isolated in $74(1.2 \%)$ samples. S. flexneri was the most common serogoup identified followed by S. boydii and S. sonnei. Yearwise isolation of Shigella serogroups was $1.58 \%$ in 2009 which decreased to $0.38 \%$ in 2013. All Shigella serogroups showed higher sensitivity to ciprofloxacin as compared to nalidixic acid, cotrimoxazole and ampicillin. Interpretation and conclusion: There is a significant increase in resistance to several commonly used antimicrobial agents. The rapid increase in ciprofloxacin resistance, especially in S. flexneri, is a major cause of concern. The results suggest reconsideration of the empiric use of these antimicrobial agents for the treatment of shigellosis.
\end{abstract}

Keywords: Antimicrobial resistance, diarrhea, Shigella

\section{INTRODUCTION}

Diarrheal diseases are an important cause of morbidity and mortality in developing countries. Of the bacterial causes of dysentery, Shigella serogroups are the major enteropathogens with outbreak potential and common development of antimicrobial resistance. ${ }^{[1]}$ Among diarrheagenic agents, Shigella should be emphasized because of its prevalence and the severity of the associated disease, accounting for 140 million cases globally per year and 60,000 deaths annually of which

Corresponding Author: Prof. Veenu Gupta

E-mail: vsunilgupta@rediffmail.com

Received: 27-07-2015

Accepted: 26-10-2015

How to cite this article: Gupta V, Kaur D, Aggarwal M, Kaur J, Chawla P, Chaudhary J. Changing resistance pattern of Shigella isolates over a period of five years. J Gastrointest Infect, 2015; 5:38-41.
$60 \%$ occur in children below 5 years of age. ${ }^{[2]}$ Recent reports from certain Asian countries show incidence rates of shigellosis ranging between $3-13 \% \cdot{ }^{[3]}$ Intestinal infection with Shigella serogroups can be managed by rehydration therapy; however, treatment with antimicrobial agents has been proven effective in reducing intensity and duration of symptoms and also in preventing lethal complications. ${ }^{[4]}$ Antimicrobial therapy is advocated for shigellosis to shorten the duration of illness. However, in Asia and Africa, antimicrobial resistance is an emerging problem among Shigella serogroups and treatment options are becoming limited globally. ${ }^{[5]}$ Shigella sp. were susceptible to many antimicrobials initially, but have developed resistance to them over a period of time. Antimicrobial susceptibility patterns of Shigella sp. vary among its serogroups and also between geographical areas periodically. ${ }^{[6]}$ A local knowledge of the distribution of Shigella serogroups, 
with their changing drug resistance patterns to guide empiric antimicrobial therapy is imperative in controlling shigellosis. Hence the present study was conducted to know the changing prevalence and antimicrobial susceptibility pattern of shigellosis over a period of five years.

\section{MATERIALS AND METHODS}

This was a retrospective study done over a period of 5 years. Stool samples were collected from cases of dysentery and diarrhea in all age groups from Jan 2009 to Dec 2013. The specimens were processed and inoculated on MacConkey's agar, deoxycholate citrate agar (DCA) and in selenite F enrichment broth. After 18-24 h, subculture was done on DCA. The plates were incubated aerobically at $37^{\circ} \mathrm{C}$ overnight. Non-lactose fermenting colonies were identified on the basis of various biochemical tests and serologically Shigella was confirmed by specific antisera (Denka Seiken, Japan). The susceptibility of all the isolated Shigella to different antibiotics was determined by Kirby-Bauer's disk diffusion technique as per the Clinical and Laboratory Standards Institute (CLSI) guidelines. ${ }^{[7]}$ The antibiotics used were ampicillin $(10 \mu \mathrm{g})$, cotrimoxazole $(1.25 /$ $23.75 \mu \mathrm{g})$, ciprofloxacin $(5 \mu \mathrm{g})$ and nalidixic acid $(30 \mu \mathrm{g})$. Antibiotic susceptibility between different Shigella serogroups was compared and trends of drug resistance to various antimicrobial agents over a period of five years was seen. Institutional Ethical Committee approval was obtained.

\section{RESULTS}

Of a total of 6117 stool samples received over a period of five years. Shigella serogroups were identified in $74(1.2 \%)$ of them S. flexneri $(93.2 \%)$ was the most common serogroup followed by S. boydii $(4.05 \%)$ and S. sonnei (2.7\%). Yearwise isolation of Shigella serogroups was $1.58 \%$ in $2009,2.01 \%$ in 2010 which decreased to $1.6 \%$ in $2011,0.61 \%$ in 2012 and $0.38 \%$ in 2013 (Table 1 ).

Table 1

Yearwise distribution of Shigella serogroups

\begin{tabular}{|c|c|c|c|c|c|}
\hline Year & $\begin{array}{c}\text { Total } \\
\text { samples }\end{array}$ & $\begin{array}{c}\text { S. flexneri } \\
\mathbf{( n = 6 9 )}\end{array}$ & $\begin{array}{c}\text { S. sonnei } \\
\mathbf{( n = 2 )}\end{array}$ & $\begin{array}{c}\text { S. boydii } \\
\mathbf{( n = 3 )}\end{array}$ & $\begin{array}{c}\text { Total (\%) } \\
\mathbf{( 7 4 )}\end{array}$ \\
\hline $\mathbf{2 0 0 9}$ & 1202 & 19 & - & - & $1.58 \%$ \\
\hline $\mathbf{2 0 1 0}$ & 1140 & 21 & - & 2 & $2.01 \%$ \\
\hline $\mathbf{2 0 1 1}$ & 1187 & 18 & 1 & - & $1.6 \%$ \\
\hline $\mathbf{2 0 1 2}$ & 1294 & 6 & 1 & 1 & $0.61 \%$ \\
\hline $\mathbf{2 0 1 3}$ & 1294 & 5 & - & - & $0.38 \%$ \\
\hline
\end{tabular}

Table 2

Percentage of Shigella serogroups resistant to antimicrobial agents

\begin{tabular}{|c|c|c|c|}
\hline Antimicrobial agents & $\begin{array}{c}\text { S. flexneri } \\
(\mathbf{n}=\mathbf{6 9})\end{array}$ & $\begin{array}{c}\text { S. boydii } \\
(\mathbf{n}=\mathbf{3})\end{array}$ & $\begin{array}{c}\text { S. } \text { sonnei } \\
(\mathbf{n}=\mathbf{2})\end{array}$ \\
\hline Ampicillin & $46(66.6 \%)$ & $1(33.3 \%)$ & $1(50 \%)$ \\
\hline Cotrimoxazole & $54(78.3 \%)$ & $2(66.6 \%)$ & $2(100 \%)$ \\
\hline Ciprofloxacin & $18(26.1 \%)$ & $0(0 \%)$ & $0(0 \%)$ \\
\hline Nalidixic acid & $65(94.2 \%)$ & $2(66.6 \%)$ & $2(100 \%)$ \\
\hline
\end{tabular}


All Shigella serogroups showed higher sensitivity to ciprofloxacin as compared to nalidixic acid (93.2\%), cotrimoxazole $(78.3 \%)$ and ampicillin $(64.8 \%)$ where high degree of resistance was observed (Fig.1) S. sonnei showed high resistance to nalidixic acid and cotrimoxazole whereas S. flexneri showed high resistance to ampicillin as compared to other serogroups (Table 2) Over the 5 year period, resistance to ampicillin increased from $42.4 \%$ in 2009 to $80 \%$ in 2013 and to ciprofloxacin from $15.8 \%$ to $40 \%$ (Fig. 2). Of the Shigella isolates, $81.5 \%$ were multidrug resistant, being commonly resistant to nalidixic acid and cotrimoxazole.

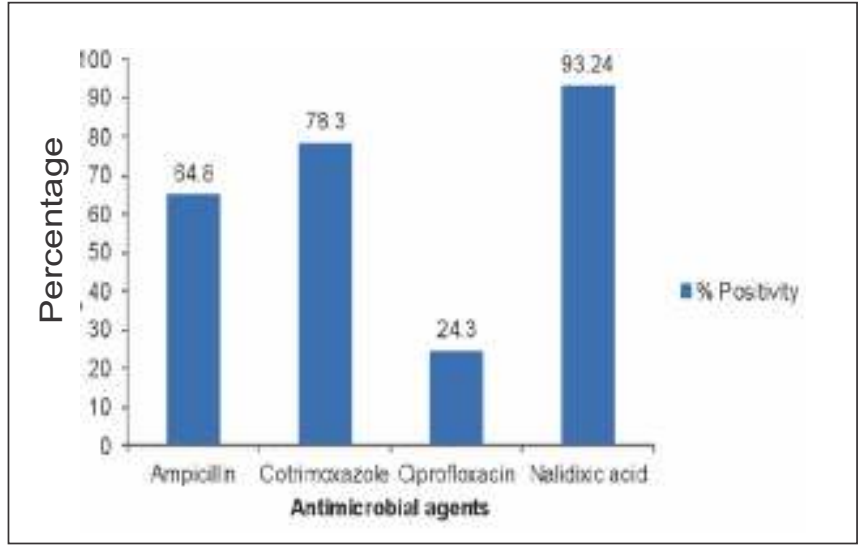

Fig.1: Antimicrobial resistance profile of Shigella isolates

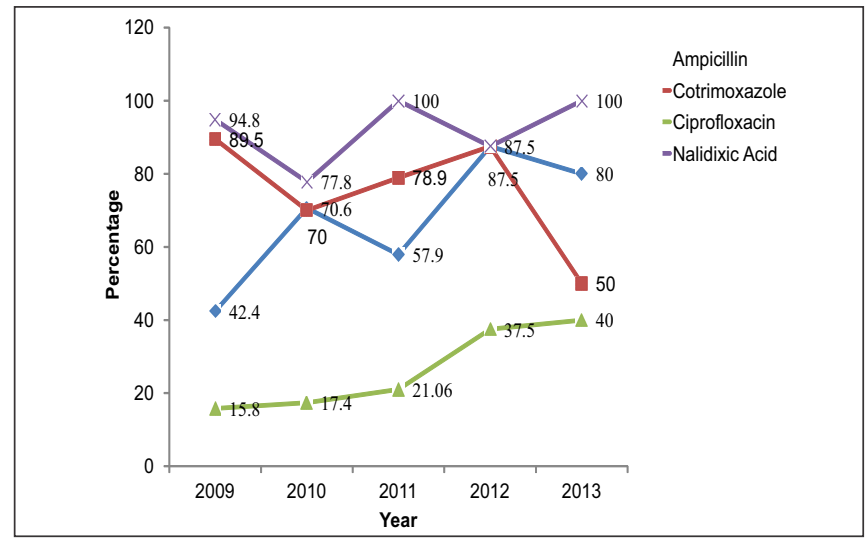

Fig.2: Percentage resistance of Shigella isolates to various antimicrobial agents (2009-2013)

\section{DISCUSSION}

Shigellosis still accounts for a significant proportion of morbidity and mortality, especially in developing countries ${ }^{[8]}$ In the present study, Shigella sp. were isolated from $1.2 \%$ stool samples. This rate is similar to studies done by Kumar et $a l^{[8]}$ and Mandal ${ }^{[9]}$ whereas higher incidence of $6.47 \%$ was reported by Tejashree et al. ${ }^{[10]}$ Rate of Shigella isolation has decreased from 2011 onwards. This difference could be due to improved public health measures, and continuing efforts by the health authorities to improve sanitation.

The pattern of shigellosis indicates that S. flexneri (93.2\%) was the most commonly isolated serogroup. This is comparable to the serogroup distribution seen in India and other developing countries ${ }^{[9,11-12]}$ where S. flexneri was the commonest whereas Rahbar et al ${ }^{[13]}$ showed S. sonnei as the commonest Shigella isolate.

Antimicrobial therapy is recommended for shigellosis because it can shorten the severity and duration of illness, reduce shedding of the organism, and prevent secondary complication and death. However antimicrobial resistance occurred among Shigella spp, since the 1940s, when sulfonamide resistance was first recognized in Japan. ${ }^{[14]}$ The present study showed high resistance to nalidixic acid (93.2\%) followed by co-trimoxazole (78.3\%) and ampicillin $(64.8 \%)$. Similar resistance pattern to nalidixic acid $(100 \%)$ and cotrimoxazole $(76.9 \%)$ was observed by Patil et al. ${ }^{[15]}$ Resistance pattern of ampicillin and cotrimoxazole observed by Kumar et $a l^{[8]}$ was comparable to our study. In contrast lower resistance to nalidixic acid (51\%) was observed by Mandal et al ${ }^{[9]}$ and to cotrimoxazole in a study from Mysore. ${ }^{[10]}$

Fluoroquinolones are recommended as the drug of choice for shigellosis by the World Health Organization. ${ }^{[8]}$ The emergence of fluoroquinolone resistant Shigella may be due to overuse of these drugs as they are empirically used for many infections like diarrhea and urinary tract infection. With the emergence of high resistance to fluoroquinolones, therapeutic options are limited. The overall incidence of resistance to ciprofloxacin was $24.3 \%$, which can be compared with other study done in Punjab showing a resistance of $13.3 \%{ }^{[8]}$ However a very high resistance to ciprofloxacin has been reported from other parts of India. ${ }^{[9,11]}$

Over the five years, resistance to nalidixic acid was the maximum and least to ciprofloxacin. Resistance to ampicillin and cotrimoxazole was comparably the same in 2010 and 2012. A similar resistance pattern was reported by Tejashree et $_{\text {al }}{ }^{[10]}$ whereas higher resistance 
to cotrimoxazole compared to ampicillin was reported by Kumar et al ${ }^{[8]}$ Of the Shigella isolates, $81.5 \%$ were multidrug resistant. The most common multidrug resistance pattern was to nalidixic acid and cotrimoxazole. Similar rate of multiple drug resistant Shigella was seen in other studies. ${ }^{[8,13]}$

In conclusion, the present study demonstrates that S. flexneri is the predominant serogroup. There is a significant increase in resistance to several commonly used antimicrobial agents. The rapid increase in ciprofloxacin resistance, especially in S. flexneri, is a major cause of concern. The results suggest reconsideration of the empiric use of these antimicrobial agents for the treatment of shigellosis.

SOURCE OF FUNDING: Institutional funds

\section{CONFLICT OF INTEREST: Nil}

\section{REFERENCES}

1. Naik DG. Prevalence and antimicrobial susceptibility patterns of Shigella species in Asmara, Eritrea, northeast Africa. J Microbiol Immunol Infect. 2006;39:392-5.

2. Jomezadeh $\mathrm{N}$, Babamoradi $\mathrm{S}, \underline{\text { Kalantar }} \mathrm{E}$ and Javaherizadeh $\mathrm{H}$. Isolation and antibiotic susceptibility of Shigella species from stool samples among hospitalized children in Abadan, Iran. Gastroenterol Hepatol Bed Bench. 2014;7:218-23.

3. Von Seidlein L, Kim DR, Ali M, Lee H, Wang X, Thiem VD et al. A multicentre study of Shigella diarrhoea in six Asian countries: disease burden, clinical manifestations, and microbiology. Plos Med. 2006;3:e353.

4. Peirano G, Souza FS, Rodrigues DP. Frequency of serovars and antimicrobial resistance in Shigella spp. from Brazil. Mem Inst Oswaldo Cruz. 2006;101:245-50.

5. Pazhani GP, Niyogi SK, Singh AK, Sen B, Taneja N, Kundu M et al. Molecular characterization of multidrug-resistant Shigella species isolated from epidemic and endemic cases of shigellosis in India. J Med Microbiol. 2008;57:856-63.

6. Bhattacharya D, Sugunan AP, Bhattacharjee $H$, Thamizhmani $R$, Sayi DS, Thanasekaran $\mathrm{K}$ et al. Antimicrobial resistance in Shigella: Rapid increase and widening of spectrum in Andaman Islands, India. Indian J Med Res. 2012;135:365-70.

7. Clinical and Laboratory Standards Institute. Performance Standards for Antimicrobial Susceptibility Testing; TwentySecond Informational Supplement. CLSI document M100S22.Clinical and Laboratory Standards Institute, Wayne, Pennsylvania USA; 2012.

8. Kumar A, Oberoi A, Alexander V. Prevalence and antimicrobial susceptibility patterns of Shigella in stool samples in a tertiary healthcare hospital of Punjab. CHRISMED J Health Res. 2014;1:33-5.

9. Mandal J1, V G, Emelda J, S M, Parija SC. The recent trends of shigellosis: A JIPMER perspective. J Clin Diagn Res. 2012;6:1474-7.

10. Tejashree A, Vijay Kumar GS, Rao R, Mahale RP, Gopalakrishnan $\mathrm{R}$, Ponna $\mathrm{Y}$. Spectrum of enteric pathogens in a tertiary care hospital Transworld Medical Journal. 2013;1:69-73.

11. Ballal M, Devadas S, Chakraborty R, Shetty V. Emerging trends in the etiology and antimicrobial susceptibility pattern of enteric pathogens in rural coastal india. International $\mathrm{J}$ of Clinical Medicine. 2014;5:425-32

12. Moez-Ardalan K, Zali.MR, Dallal MM, Hemami MR, Salmanzadeh-Ahrabi S. Prevalence and Pattern of antimicrobial resistance of Shigella species among patients with acute diarrhea in Karaj Tehran, Iran. J Health Popul Nutr. 2003;21:96-102.

13. M Rahbar, M Deldari, M Hajia. Changing prevalence and antibiotic susceptibility patterns of different shigella species in Tehran, Iran. The Internet Journal of Microbiology. 2006;3:1-4.

14. Lima AM, Lima.NL, Pinho MN, Barros EA, Teixeira MJ, Martins $\mathrm{MV}$ et al. High frequency of strains multiply resistant to ampicillin, trimethoprim-sulfamethoxazole, streptomycin, chloramphenicol and tetracycline isolated from patients with shigellosis in Northeastern Brazil during the period 1988-1993. Antimicrobiol Agents and Chemotherapy. 1995;39:256-9.

15. Patil D, Lava R. Identification, characterization and antibiotic suceptibility of Shigella species isolated from stool samples in children. Int J Biol Med Res. 2012; 3:1640-3. 